Etica \& Politica / Ethics \& Politics, 2005, 2

http://www.units.it/etica/2005_2/PALONEN.htm

\title{
Max Weber als Begriffspolitiker
}

$\underline{\text { Kari Palonen }}$

Department of Social Science and Philosophy

University of Jyväskylä

\begin{abstract}
Max Weber's well-known distinction between science and politics should not prevent us from recognising that his view of scholarly activity has resemblance to politics in several respects. Both refer to forms of contingent and controversial striving for power shares and their redistribution, and in so far is the scholarly activity part of politics. This does not hold only for the struggles for academic power shares but also for the contest between theories and approaches among scholars. Weber's own use of concepts for contesting or legitimising standpoints or perspectives is worth discussing. Weber uses different rhetorical strategies. Occasionally he refers to a seemingly shared sense of a concept but subverts the apparent consensus and advocates an entirely different view. For example when Weber defends 'objectivity' this does not refer to the object-dependency of research but to the fair competition between different perspectives of interpreting the object. He also uses rhetoric of provocative laughter, for example against the politically dilettantish "ink bottle" literati. Demagogy is a concept that Weber uses in depreciating, neutral or appreciating tone depending on the context. Also in his academic politics Weber prefers the politician to the official.
\end{abstract}

Es ist ein Gemeinplatz, dass Max Weber zwischen Wissenschaft und Politik streng unterscheidet. Die Grundidee seiner Wertfreiheitsthese ist, dass unter Berufung auf Wissenschaft keine Politik betrieben werden, von ihr „abgeleitet” oder auch nur in deren Namen legitimiert werden kann. „Eine empirische Wissenschaft vermag niemanden zu lehren, was er soll, sondern nur, was er kann und - unter Umständen was er will", heißt es im Objektivitätsaufsatz (Weber 1904, 151). Dies bedeutet vor allem die Autonomie der Politiker hinsichtlich einer Bevormundung durch die Wissenschaft, deren Autonomie Weber wiederum gegen Eingriffe der Regierung und anderer Mächte verteidigt.

\section{Politik und Wissenschaft treiben}

Das Verhältnis von Wissenschaft und Politik bei Weber kann man jedoch auch von der performativen Seite der Tätigkeiten aus analysieren. Studiert man die Art der Handlungen der Akteure, dann werden weder Wissenschaft noch Politik als Lebenssphären verstanden. Vielmehr fragt man: was tut dann jemand, der Politik bzw. Wissenschaft treibt? 
Weber hat die performative Seite der Wissenschaftler nie eigenständig behandelt, während er dies hinsichtlich der Politiker explizit tat. Meiner These nach bildet der Webersche Idealtypus des Politikers auch ein Modell für die Tätigkeiten des Wissenschaftlers. Auch in der Wissenschaft geht es - um nur die berühmtesten der Weberschen Politik-Formeln zu notieren - zumindest bis zu einem gewissen Grade um das "Streben nach Machtanteilen und nach Beeinflussung der Machtverteilung" (Weber 1919, 36), um "langsames Bohren harter Bretter mit Leidenschaft und Augenmaß zugleich" (ebenda, 88), um "Parteinahme, Kampf, Leidenschaft" (ebenda, 53) oder um "Kampf, Werbung von Bundesgenossen und von freiwilliger Gefolgschaft"(1918a, 232),

Gegen den weit verbreiteten akademischen Anspruch, unpolitisch zu sein, kann man folgende Einsicht Carl Schmitt zitieren: "In Wahrheit ist es ... eine typische und besonders intensive Art und Weise, Politik zu treiben, dass man den Gegner als politisch, sich selbst als unpolitisch (d.h. hier wissenschaftlich, gerecht, objektiv, unparteiisch usw.) hinstellt" (Schmitt 1932, 21). Unabhängig davon, dass Schmitts Entpolitisierung für Weber eine Politisierung ist (s. Palonen 1998, 226-229), lohnt es sich, das Politikum auch in den Praktiken des Wissenschaftsbetriebes aufzusuchen.

Entsprechend kann man den akademischen Betrieb als ein Spielfeld der Theoriepolitik verstehen (zu diesem Begriff s. Palonen 2003a, 68-69). Im Streit unter Gelehrten geht es nicht nur um Stellen, Gehaltsgrade, Forschungsgelder, Mitarbeiter, Lehr- und Lernfreiheit, Zeit für Forschung, Publikationen, akademische Prestige u.a. äußere Aspekte des Betriebs, die unter Professoren seit jeher zu leidenschaftlichen Kämpfen geführt haben. Es geht vielmehr auch um den akademischen Inhalt, um die Ansätze, Theorien und Begriffe, um ihre Konstruktion und Revision, Auf- und Abwertung, Legitimation und Delegitimation usw. Ohne Politik und damit verbundene Rhetorik kommt keine Theorie auf und kann auch keine bekämpft werden.

Das Politikum des akademischen Betriebs ist in der perspektivistischnominalistischen Wissenschaftskonzeption Max Weber schon eingebaut. Der Streit um die grundlegenden Bewertungen und Annahmen, die immer einen Bezug zur Politik haben, gehört zum Kern der Kulturwissenschaften selbst. „Das Kennzeichen des sozialpolitischen Charakters eines Problems ist es ja geradezu, daß es nicht auf Grund bloß technischer Erwägungen aus feststehenden Zwecken heraus zu erledigen ist, daß um die regulativen Wertmaßstäbe selbst gestritten werden kann und muß, weil das Problem in die Region der allgemeinen Kulturfragen hineinragt", heißt es schon im Objektivitätsaufsatz (Weber 1904, 153).

Wenn ich Weber als eine Art frühen Vertreter der Begriffsgeschichte identifiziere (Palonen 2000 und 2004), dann stellt sich die Frage, wie Max Weber selbst mit Begriffen Politik betreibt? Gelten seine für Politiker präsentierten Kriterien auch für seine eigene Begriffspolitik? War er als Begriffspolitiker erfolgreich? Welche rhetorische Strategien und Taktiken (im Sinne von Skinner 1974, revidiert in 2002) 
benutzt er für die eigene Begriffsbildung? Wie sieht man das Politikerparadigma des Wissenschaftlers in Webers eigenem Begriffsgebrauch?

\section{Webers akademischer Pyrrhussieg}

Max Weber war ein akademischer Mehrkämpfer, der in der heutigen akademischen Welt fast undenkbar geworden ist. Mag sein, dass dies auch ein Grund dafür war, dass sein Werk unter den schon zunehmend spezialisierten Zeitgenossen zwar Anerkennung genoss, aber weder ordentlich rezipiert noch gut verstanden wurde. Insofern fragt man sich, in wieweit der spätere weltweite akademische Erfolg und die dementsprechende Kanonisierung zum Klassiker dem Weberschen Streben nach akademischen Machtanteilen entspricht.

Insofern Weber nach dem Zweiten Weltkrieg zu den meist zitierten Klassikern gehört, dann kann man mit guten Gründen von einem posthumen Pyrrhussieg Webers in der anglophonen Soziologie und Politikwissenschaft sowie in ihrer europäischen Rezeption sprechen. In dem was Soziologen (von Talcott Parsons bis C.Wright Mills) und Politologen (z.B. David Easton und Robert Dahl) etwa von Politik, Macht und Herrschaft sagen, sind durchaus Spuren von Weber zu finden, aber derart verwässert, dass man von einem Vulgär-Weber sprechen muss. In den „Definitionen” dieser Begriffe sieht man Formeln, die entweder explizit auf Weber bezogen sind oder Weber-Spezialisten bekannt vorkommen. Trotzdem enthalten die Sprechakte, in denen die Begriffe verwendet werden, einen Ton, der Weber fremd ist, der ihn vereinfacht und verflacht.

Wenn etwa Politik als "Streben nach der Macht” in irgendeiner englischer Formulierung auftaucht, vergisst man entweder den Chancencharakter der Macht oder gibt dafür eine Übersetzung - etwa probability, ability or capacity -, die sowohl die radikale Kontingenz als auch das Politikum des Weberschen Chancenbegriffs verschwinden oder harmlos erscheinen lassen.

Wenn man die Rezensionen von Politik als Beruf nach der ersten (1919) und der zweiten, posthumen (1926) Auflage liest, wird klar, dass die Rezensenten kein Verständnis für die Außerordentlichkeit der kleinen Schrift zeigen. In dieser Hinsicht besteht ein deutlicher Kontrast zu Carl Schmitts Begriff des Politischen (Artikelversion 1927, Buchversion 1932), der sofort eine breite, hauptsächlich kritische aber teilweise auch enthusiastische Resonanz erhielt.

Man kann darüber streiten, ob schon Politik als Beruf oder erst Der Begriff des Politischen einen Wendepunkt in der Begriffsgeschichte der Politik bezeichnen (s. Palonen 2002, 19-21). Ich und andere, die auf Weber setzen, nehmen fast immer auch auf Schmitt Bezug, die heutigen Schmitt-Zitierer - etwa Chantal Mouffe in ihrer jüngsten Schrift On the Political (2005) - erwähnen dagegen Weber überhaupt nicht. Offenbar bleibt die Webersche Orientierung auf die Praxis der Politik aus ihrer Sicht trivial, während die Freund-Feind-Unterscheidung Schmitts, wie es bei Mouffe $(2005,8)$ heißt, auf die „Ontologie” des Politischen verweist. Ein 
Grund dürfte auch darin liegen, dass die Schmitt-Rezeptionen nur den textbookWeber kennen und keinen Bezug auf die Weberologie der letzten zwanzig Jahre nehmen.

Sowohl der vulgäre Weber der Sozialwissenschaftler als auch der triviale Weber der Schmittianer verweisen auf die Erfolglosigkeit Webers als akademischer Begriffspolitiker. Seine Stichworte sind Gemeinplätze geworden, die innovativen Aspekte seiner Begriffe sind in der breiteren Rezeption aber verloren gegangen. Dies erinnert fast an Webers Kandidatur für die Weimarer Nationalversammlung im Wahlkreis Hessen-Nassau, bei der er auf einen hinteren Listenplatz gesetzt wurde. Insofern ist es notwendig, die impliziteren Dimensionen der Weberschen Begriffspolitik auch für die heutigen Leser sichtbar zu machen.

Denken wir nur an folgende Kurzformel in Politik als Beruf: „Wer Politik treibt, erstrebt Macht" (Weber 1919, 36). Ist dies nicht eine Allerweltsbehauptung? Vor Weber haben auch andere Ähnliches gesagt, der Rechtsphilosoph Fritz Berolzheimer etwa schrieb: „Der Wesenszug aller Politik ist Streben nach Macht durch Gestaltung des Rechts." (Berolzheimer 1907/08, 243). Sagt Weber, obgleich er auf das „Wesen” verzichtet, etwas Anderes?

Wieder geht es darum, dass Weber - und nicht andere - mit "Macht" gerade auf bestimmte Art von Chancen verweist. "Macht bedeutet jede Chance, innerhalb einer sozialen Beziehung den eigenen Willen auch gegen Widerstreben durchzusetzen, gleichviel worauf diese Chance beruht", lautet seine berühmte Formel (Weber 1922, 28). Wenn statt Macht Machtchancen angesprochen werden, wird deutlicher, dass "Macht" bei Weber auf keine Totalität, sondern auf Handlungen bezogen ist, und "Chancen" - wie Weber in seiner längeren Politikformel sagt - aus bestimmten einzelnen Machtanteilen und ihrer "Verteilung” bestehen. Mit anderen Worten: „Macht” ist kein Besitz und auch keine bloße asymmetrische Relation von Über- und Unterordnung, sondern alle politischen Akteure haben irgendwelche Machtanteile, und es kommt auf die situationelle Bedeutung dieser verschiedenen Machtanteile an. Dies fehlt beim textbook-Weber gänzlich.

Nun kann man das oben zitierte Schmitt-Diktum mit Weber präzisieren. Wenn eine Person sagt, dass sie nicht nach Macht strebe, kann dies aus Webers Sicht zweierlei bedeuten: Entweder hat sich die Person eben von der Politik verabschiedet, d.h. sie ist vom Gelegenheitspolitiker zum freiwilligen Untertanen übergegangen, oder sie versucht den Sprechakt „Ich strebe nicht nach Macht” gerade in einen Machtanteil der eigenen Politik zu verwandeln, d.h. die bekannte Rolle des Antipolitikerpolitikers zu spielen.

Mit den neueren Übersetzungen haben auch die anglo- und frankophonen Weberologen jetzt bessere rhetorische Mittel als Weber selbst, die Weberschen Begriffe - und nicht nur seine Stichworte - in der akademischen Welt detailliert und historisch genug auszulegen. Vielleicht kann man hier von einem "langsamen Bohren harter Bretter" sprechen. 


\section{Die Umformulierung der Begriffe}

Um die Webersche Begriffspolitik zu verstehen, sollte man auf die Rezeptionsgeschichte lieber verzichten und Webers Begriffsbestimmungen als politische Sprechakte oder rhetorische Schachzüge in ihrem zeitgenössischen Kontext verstehen. Dieser Kontext kann nie bloß akademisch sein, vielmehr spielt dabei Webers Haltung zum deutschen Kaiserreich und zur jeweils aktuellen politischen Situation durchaus mit.

Das Beispiel des Machtstrebens verweist schon auf eine bestimmte rhetorische Strategie Webers. Mit Absicht erweckt er Eindruck einer breiten Zustimmung, und zwar zu einem Standpunkt, der keineswegs selbstverständlich ist, und bringt dadurch ein eigenes Profil ins Detail der Formulierung. In Politik als Beruf fragt Weber anfangs: „Was verstehen wir unter Politik?” (Weber 1919, 35). Wer bildet in diesem Satz das 'Wir'? Weber verweist dann auf einige allägliche Gebrauchsweisen des Begriffs: „Der Begriff ist außerordentlich weit und umfasst jede Art selbständig leitender Tätigkeit. Man spricht von der Devisenpolitik der Banken, von der Diskontpolitik der Reichsbank, von der Politik einer Gewerkschaft in einem Streik..." (Weber 1919, 35). Trotzdem liegt seine Pointe eben in der eigenständigen Umprägung des Begriffs.

Mit anderen Worten, das "Wir” bei Weber ist ein Pluralis majestatis in einem spezifischen Sinn (s. auch Palonen 2002, 25-26). Die Berufung auf das "Wir“ dient Weber dazu, seine Konzeption durch eine Anknüpfung an den Alltagsgebrauch stärker zu legitimieren (s. Skinner 1974 über den innovativen Ideologen). Bei einem Vergleich mit der zeitgenössischen Diskussion des Politikbegriffs wird klar, dass gerade Weber sich mit dem Begriff viel ausführlicher als andere seiner Zeitgenossen befasst und mit den unterschiedlichen Nuancen der Formulierung gerade ein eigenständiges Profil schafft. Man fragt sich jedoch, ob er die Singularität sowohl seiner Fragestellung als auch seiner Antworten selbst unterschätzte? Oder geht es vielmehr darum, dass Weber in seinem eigenen Werk Eitelkeit, die "Todessünde" des Politikers und die „Berufskrankheit” des Akademikers (Weber 1919, 74), streng vermeiden wollte?

Noch deutlicher kann man diese Fragen hinsichtlich der „Vorbemerkung” zu seinen Soziologischen Grundbegriffen stellen. Weber schreibt nämlich: «Vorbemerkung. Die Methode dieser einleitenden, nicht gut zu entbehrenden, aber unvermeidlich abstrakt und wirklichkeitsfremd wirkenden Begriffsdefinitionen beansprucht in keiner Art: neu zu sein. Im Gegenteil wünscht sie nur, in - wie gehofft wird zweckmäßigerer und etwas korrekterer (eben deshalb freilich vielleicht pedantisch wirkender) Ausdrucksweise zu formulieren, was jede empirische Soziologie tatsächlich meint, wenn sie von den gleichen Dingen spricht. Dies auch da, wo scheinbar ungewohnte oder neue Ausdrücke verwendet werden. Gegenüber dem Aufsatz S. 427 ff. dieses Bandes ist die Terminologie tunlichst vereinfacht und daher auch mehrfach verändert, um möglichst leicht verständlich zu sein. Das 
Bedürfnis nach unbedingter Popularisierung freilich wäre mit dem Bedürfnis nach größtmöglicher Begriffsschärfe nicht immer vereinbar und muß diesem gegebenenfalls weichen. (Weber 1922, 1)

Wenn man die "Grundbegriffe" dann liest, erscheint die zitierte Formel als ein extremes Understatement, das rhetorisch auf eine höhere Akzeptanz der Weberschen Begriffsumbildungen zielt. Gewissermaßen gegen die lexikalischen Traditionen der Reihe Grundrisse der Sozialökonomik, unternimmt Weber bewusste Umprägungen der Begriffe. Er analysiert „die gesellschaftlichen Ordnungen und Mächte”, wie es im Untertitel heißt, nicht als „Fakten”, nicht als „Gesetzmäßigkeiten” oder auch nicht als normative Forderungen, sondern strikt nominalistisch, als Komplexe von "Chancen”. Heute würde man diesen Unterschied vielleicht als Anspruch auf einen Paradigmenwechsel bezeichnen.

Die Chancen, das heißt: das Mögliche, das Kontingente, das Erwartbare, das Realisierbare, die Gelegenheiten, das Bespielbare - wie immer man die unterschiedlichen Facetten dieses mehrschichtigen Schlüsselbegriffs bei Weber auslegt (s. Palonen 1998) - sind nach Weber für die handelnden Akteure etwas Reales. Dass er etwa den Staat als einen bestimmten Komplex von Herrschaftschancen bestimmt, enthält schon eine Absage an die personifizierende Fiktion - „General Doktor vom Staat”, wie es bei Thomas Manns Betrachtungen eines Unpolitischen heißt (Mann 1918). Der Staat bei Weber verweist auf die Handlungen der Personen, die im seinem Namen bzw. gegen die im Namen des Staates tätigen Personen agieren: „Ein »Staat« hört z.B. soziologisch zu »existieren" dann auf, sobald die Chance, daß bestimmte Arten von sinnhaft orientiertem sozialem Handeln ablaufen, geschwunden ist" (Weber 1922, 13).

Wenn Weber etwa Herrschaft - „Herrschaft soll heißen die Chance, für einen Befehl bestimmten Inhalts bei angebbaren Personen Gehorsam zu finden” (ebenda, 28) - als Chance bestimmt, dann ist dies eben keine bloße Umformulierung der Alltagssprache. Vielmehr betreibt Weber damit eine absichtliche Auflösung der alläglichen Kollektivbegriffe (s. Weber 1904, 210-212). „Herrschaft” ist für Weber nie ein faktischer Tatbestand, sondern verweist auf das Mögliche, dessen „Realisierungsgrad" von den Handlungsmöglichkeiten und der Wahl zwischen diesen unter den zum Gehorsam Bestimmten abhängig ist. Chancen zur Verweigerung des Gehorsams, die unterschiedliche Arten und Grade von abschätzbaren Risiken enthalten, gibt es jederzeit. Die Frage ist vielmehr: welche Arten von Chancen vorliegen, mit welchen erwartbaren und denkbaren Folgen in ihrem Zusammenhang zu rechnen ist. Es steht den zum Gehorsam Bestimmten frei, die Chancen und die damit immer verbundenen Nebenfolgen - d.h. diejenigen Chancen, die im Augenblick des Handelns nicht thematisiert wurden - des Gehorsams bzw. der Verweigerung desselben selbständig abzuschätzen und zwischen ihnen zu wählen.

Webers Umdeutung der aus „sozialem Handeln” und „sozialen Beziehungen” entstehenden „Ordnungen und Mächte” aus der Sicht der „Chancen” bedeutet die Anerkennung der Omnipräsenz der Kontingenz. Damit ist natürlich nicht eine Welt 
ohne Zwänge, Bedrängnisse u.a. gemeint, sondern eben eine, in denen diese als absichtliche Resultate bzw. als Nebenfolgen kontingenter Handlungen entstehen und wieder aufgelöst werden können. Für eine derartige Welt brauch man nicht nur eine andere Politikwissenschaft als die der Vulgär-Weberianer, sondern auch die Einsicht in das Politikum der Wissenschaft im Allgemeinen.

Der Personentypus, der den Chancencharakter der menschlichen Beziehungen und die aus ihnen entstehenden "Ordnungen und Mächte" am ehesten versteht, ist es eben der Politiker. Im letzten Abschnitt von Politik als Beruf wird dieser Art von ,Möglichkeitsmensch' als einer beschrieben, der eine Kombination von entgegengesetzten Anforderungen beherrschen kann: „Es ist ja durchaus richtig, und alle geschichtliche Erfahrung bestätigt es, daß man das Mögliche nicht erreichte, wenn nicht immer wieder in der Welt nach dem Unmöglichen gegriffen worden wäre. Aber der, der das tun kann, muß ein Führer und nicht nur das, sondern auch - in einem sehr schlichten Wortsinn - ein Held sein" (Weber 1919, 88; auch Weber 1917a, 514). In einem ähnlichen Sinn sagt Weber schon 1906 in einer Polemik gegen Eduard Meyers Kritik an Spekulationen in der Geschichtswissenschaft, dass der Historiker die Geschichte wie der handelnde Mensch - d.h. wie ein Politiker - behandeln sollte, d.h. er sollte mit offenen Möglichkeiten operieren (s. Weber 1906, 267).

\section{Die Umprägung der 'Objektivität'}

Neben dem Chancencharakter der Begriffe zeigt sich in der kompromisslosen Anerkennung der Perspektivität jeder Erkenntnis die zweite Leitidee der Weberschen Wissenschaftstheorie: „Es gibt keine schlechthin »objektive“ wissenschaftliche Analyse des Kulturlebens oder ... der "sozialen Erscheinungen" unabhängig von speziellen und »einseitigen« Gesichtspunkten, nach denen sie ausdrücklich oder stillschweigend, bewußt oder unbewußt - als Forschungsobjekt ausgewählt, analysiert und darstellend gegliedert werden." (Weber 1904, 170). Diese Sicht verweist durch Nietzsche historisch auf die klassische Einsicht der antiken Rhetoriker und Sophisten, dass nämlich Phänomene nicht von den Perspektiven der Interpretation loszulösen sind, dass die sprachliche Formulierung der Perspektive vielmehr zum Verständnis des Phänomens gehört.

Weber spricht immer von der Pluralität und Konkurrenz der Perspektiven, und zwar nicht im Sinne eines Residuums, sondern eben als Vorrausetzung der Erkenntnis überhaupt: «Alle Erkenntnis der Kulturwirklichkeit ist, wie sich daraus ergibt, stets eine Erkenntnis unter spezifisch besondert en Gesichtspunkten. Wenn wir von dem Historiker und Sozialforscher als elementare Voraussetzung verlangen, $\mathrm{da} ß$ er Wichtiges von Unwichtigem unterscheiden könne, und daß er für diese Unterscheidung die erforderlichen "Gesichtspunkte« habe, so heißt das lediglich, daß er verstehen müsse, die Vorgänge der Wirklichkeit - bewußt oder unbewußt auf universelle "Kulturwerte« zu beziehen und danach die Zusammenhänge 
herauszuheben, welche für uns bedeutsam sind. Wenn immer wieder die Meinung auftritt, jene Gesichtspunkte könnten dem "Stoff selbst entnommen« werden, so entspringt das der naiven Selbsttäuschung des Fachgelehrten, der nicht beachtet, daß er von vornherein kraft der Wertideen, mit denen er unbewußt an den Stoff herangegangen ist, aus einer absoluten Unendlichkeit einen winzigen Bestandteil als das herausgehoben hat, auf dessen Betrachtung es ihm allein ankommt (ebenda, 180).»

Gerade hier liegt auch der Grund dafür, dass die 'Objektivität' bei Weber gerade und 'bloß' in der möglichst scharfen Konkurrenz der Perspektiven besteht. Nur deswegen ist es möglich, naive alltägliche Ansichten durch Konfrontation mit anderen zu revidieren.

Ein starker begriffspolitischer Schachzug Webers besteht also darin, etwas - in Anführungszeichen - als Objektivität zu bezeichnen, was von der gesamten akademischen Tradition explizit abweicht. Nicht nur die Zeitgenossen, sondern das gesamte Pathos der Wissenschaftsapologeten oder besser: -theologen aller Zeiten pflegten die Objektivität ja als den größten Vorzug der Wissenschaft zu predigen. Webers Politik der Umdeutung des Begriffs - anstatt Objektivität durch einen anderen Begriff zu ersetzen oder ihn zu entwerten - ist eine Folge seines Nominalismus und Perspektivismus. Das Objektive kann nicht das Objektgemäße sein, weil die Objekte „an sich” nichts „sind”, sondern erst im Zusammenhang der sie deutenden Perspektiven als etwas erscheinen.

Mit dem Festhalten am Titel Objektivität verweist Weber auf ein anderes Problem als die Objektivisten. Es geht nicht um die Selbstauflösung der Akteure unter dem Objekt: gegen diese Forderung richtet sich seine Berufung auf die „einseitige Steigerung” der Perspektiven und der damit verbundenen Begriffsapparatur des Idealtypus. Es geht Weber vielmehr darum, die Objektivität als regulatives Prinzip hinsichtlich der Chancen in der Konkurrenz zwischen den Perspektiven zu präsentieren. Wenn es keine zureichenden Gründe gibt, das Objekt selbst zum sprechen zu bringen, muss der Streit der Perspektiven so geregelt werden, dass alle Perspektiven - ob alt oder neu, ob etabliert oder marginal usw. - möglichst gleiche Chancen haben, sich zu bewähren. Wenn man also das Objekt nicht erschöpfend kennen kann, muss man sich stattdessen um die Vielseitigkeit und Erneuerungsfähigkeit der Interpretationen kümmern.

Die Konkurrenz der Perspektiven steht auch hinter zwei Aspekten der Begriffsbildung bei Max Weber, nämlich hinter der heuristische Rolle der Umstrittenheit der Begriffe (s. Palonen 2000) sowie der Ersetzung der quasidinghaften Deutung der Begriffe durch temporale, verzeitlichte Begriffe (s. Palonen 2003b). Für diese Konkurrenz der Perspektiven gibt es bei Weber auch ein klares politisches Modell, nämlich der Parlamentarismus englischer Prägung, der sowohl konzeptionell als auch historisch auf das rhetorische Prinzip des Wechsel von Rede und Widerrede zurückkehrt (s. z.B. Redlich 1905). Diese „parlamentarische” Theorie des Wissens stellt Weber explizit gegen das bürokratische Modell des 
„Sachwissens”, „Dienstwissens” und „Geheimwissens” (besonders Weber 1917b und 1918a, dazu s. Palonen 2004).

\section{Die Rhetorik der Provokation}

Die Pointe der Weberschen Berufung auf das Gängige liegt also darin, dass sie der Eigenständigkeit weite Randzonen eröffnet. Die „zweckmäßige und korrekte Ausdrucksweise" verweist darauf, dass "Sachen" für Weber von ihrer sprachlichen Formulierung abhängig sind, dass die Phänomene als solche sprachlich mitkonstruiert werden. Die Berufung auf das Gängige zielt auf die Überredung derjenigen, die zwischen Worten und Dingen streng unterscheiden und für die "bloße Worte" nur die Oberfläche der Dinge sind. Mit anderen Worten, Weber nutzt die zeitgenössische Wissenschaftsgläubigkeit bzw. den Alltagsrealismus der Öffentlichkeit geschickt, um eine eigene Profilierung bei seiner Begriffsbildung zu betreiben.

In anderen Kontexten ist seine Profilierung viel offener, er spielt sogar mit einer klar provozierenden Rhetorik. Die akademischen Kritiker haben die Webersche Pointe vielfach missverstanden bzw. seine Distanzierung vom etablierten Wortgebrauch nicht beachtet. In seltenen Fällen antwortet Weber den Kritikern: Dann veranschaulicht er die Pointe so deutlich, dass die Kritiker zugleich als Dilettanten denunziert werden. Der Paradefall hierfür stammt aus seiner Antwort an Felix Rachfahl im Jahre 1910: „Was der 'übliche Sinn' des 'kapitalistischen Geistes' ist, kümmert mich nicht, auch nicht ob das 'Tiergartenviertel' oder 'Agrarier' oder Leutnants mit üppigem Geldbeutel die meisten Aystern konsumieren" (Weber 1910, 170). Weber zu beschuldigen, sich nicht an den gängigen Wortgebrauch zu halten, ist also gerade deswegen Unsinn, weil er explizit ausgedrückt hat, in welchem spezifischen Sinn er vom „kapitalistischen Geist” spricht.

Um den Dilettantismus des Kritikers nochmals hervorzuheben, erklärt er, was er mit dem angesprochenen Begriff meint: „Sondern es kam mir bei jenem... Beispiel auf die Illustration einer sehr spezifischen innerlichen Beziehung zum Erwerb und Besitz: des Gefühls der 'Verantwortung' gegenüber dem eigenen Vermögen, welches 'irrationale' Ausgaben nicht nur ablehnt, sondern wie eine eigentümliche Art von 'Versündigung' ansieht (was mit dem gewöhnlichen Geiz, von dem Rachfahl an anderer Stelle redet, nichts zu schaffen hat). Es ist ein asketisches Bedenken gegen den Genuß als solchen." (ebenda). Eine derartige Erläuterung mag insofern selbstkritisch sein, als Weber damit zugesteht, dass vielleicht auch andere seine Pointe übersehen haben. Darüber hinaus betont Weber mit Nachdruck, dass es dann, wenn jemand wie er von einem Thema spricht, immer notwendig ist, die Fragestellungen und Formulierungen des Autors sehr genau zu beachten.

In seinen politischen Interventionen ist Weber in seinem Begriffsgebrauch viel freier, und gerade deswegen sind diese Schriften für Webers Begriffspolitik höchst 
anregend. Mein Lieblingsbeispiel für die Rhetorik der offenen Provokation ist sein Aufsatz Wahlrecht und Demokratie in Deutschland (1917), in dem er die Gegner im klassischen rhetorischen Sinn als lächerlich erscheinen lässt (s. Skinner 2001). Hier begrenze ich mich auf die Art, mit der er die zeitgenössischen akademischen und literarischen Gegner der Demokratisierung und der Parlamentarisierung in Deutschland provoziert. Sein erstes Beispiel gilt dem Pluralwahlrecht, mit dem er einleitend wie folgt zu Gericht geht: «Welches andere Wahlrecht könnte an seine Stelle treten? Bei den Literaten erfreuen sich allerhand Pluralwahlsysteme großer Beliebtheit. Welche aber? Soll der Familienstand, etwa durch Zusatzstimmen, privilegiert werden? Die Unterschichten des Proletariats und die Bauern auf den ärmsten Böden, überhaupt aber alle Schichten mit der geringsten ökonomischen Voraussicht, heiraten am frühesten und haben die meisten Kinder. Oder - der Lieblingstraum der Literaten - die "Bildung"? Unterschiede der "Bildung" sind heute, gegenüber dem klassenbildenden Element der Besitz- und ökonomischen Funktionsgliederung, zweifellos der wichtigste eigentlich ständebildende Unterschied» (Weber 1917b, 156-157).

Damit deutet Weber schon eine Schwachstelle der im Laufe des 19. Jahrhunderts eifrig geführten Debatte der politischen „Kapazität” oder „Kompetenz” an. Jede Gruppierung will die Zusatzstimmen in einer Weise bestimmen, die dem eigenen Standpunkt nutzt und dem gegnerischen schadet. Weber zeigt mit einfachen Beispielen die politisch unerwünschten Folgen derartigen Vorschläge sowie die Unmöglichkeit, sich neutrale Gründe für derartige Entscheidung auszudenken. Auch das Bildungskriterium, das seine akademischen Kollegen vor allem bevorzugen, ist, wie Weber weiter ausführt, sowohl beliebig als auch grenzenlos interpretierbar: "Soll wirklich das Examensdiplom, welchem schon die Masse aller Ämter ausgeliefert ist, und die dadurch patentierte Schicht mit ihren sozialen Prätensionen noch weiter privilegiert werden? Soll dem Pfründenhunger der examinierten Amtsanwärter - deren Zahl durch die Frequenzkonkurrenz der Hochschulen und den sozialen Ehrgeiz der Eltern für ihre Kinder ungeheuer über den Bedarf gesteigert ist - die Macht über den Staat zugewendet werden? Und was hat eigentlich der Doktor der Physik oder der Philosophie oder Philologie mit politischer "Reife" zu tun? Jeder Unternehmer und jeder Gewerkschaftsführer, der, im freien Kampf um das ökonomische Dasein stehend, die Struktur des Staates täglich am eigenen Leibe spürt, weiß mehr von Politik als derjenige, dem der Staat nur die Kasse ist, aus der er kraft Bildungspatentes eine standesgemäße, sichere, pensionsfähige Einnahme erhält» (ebenda, 157).

Hier zeigen sich nicht nur Webers zentrale politische Anliegen, nämlich die Bekämpfung der neuen feudalen Tendenzen (auf akademischer oder kapitalistischer Grundlage) sowie der auf diplomiertem Sachwissen basierte Beamtenherrschaft. Vor allem richtet sich seine Polemik gegen den politischen Dilettantismus deutscher Akademiker, die - anders als Unternehmer oder Gewerkschaftler - mit der Praxis des Kampfs persönlich nicht vertraut sind. Die Hobbypolitik der Honoratioren bleibt für Weber notwendigerweise dilettantisch, auch die der Beamten. „Das sind 
Schmarotzerideale einer Pfründner- und Rentnerschicht, welche den schweren Alltag der geistig und körperlich arbeitenden Mitbürger an ihrem Tintenfaßhorizont messen zu wollen sich erdreistet." (ebenda). Die erste Bedingung für die „politische Reife" besteht bei Weber in der Absage an die ständisch-korporativen Sonderinteressen der Akademiker.

Das „Tintenfaß" ist heute etwas Anachronistisches, das wir, die in den fünfziger und sechziger Jahren in die Schule gingen, noch kennen, das aber längst durch Kugelschreiber u.a. abgelöst wurde. Es ist eine von Weber bevorzugte Metonymie für die Enge des politischen Horizonts der deutschen Akademiker.

Die „Tintenfaßromantiker" erkennen den freiwilligen Charakter der Parteien und Organisationen nicht an (ebenda, 168), die „Tintenfaß-Ideologen” sprechen von „Gemeinwirtschaft" wenn es um kapitalistische Kartelle geht (ebenda, 171). Die These, dass „(d)as deutsche Parlament bisher große politische Talente nicht hervorzubringen vermocht (habe)", ist nach Weber ein weiterer Ausdruck dafür, dass sie das Werk der Paulskirchenparlamentarier nicht verstanden haben und „deutscher Geist” dort besser lebte als „als in den Tintenfässern dieser Herren” (1918a, 207).

Trotzdem soll das nicht als Diskreditierung des Worts als Medium verstanden werden. Vielmehr benutzt Weber die Metonymie auch für die Betonung der Rolle der Rhetorik im politischen Handeln: „Heute ist nun einmal nicht das eigene Dreinschlagen mit dem Schwert, sondern sind ganz prosaischen Schallwellen und Tintentropfen: geschriebene und gesprochene Worte, die physischen Träger des leitenden (politischen und: militärischen!) Handelns. Es kommt nur darauf an, daß Geist und Kenntnisse, starker Wille und besonnene Erfahrung diese Worte: Befehle oder werbende Rede, diplomatische Noten oder amtliche Erklärungen im eigenen Parlament formen." (ebenda, 237).

Diese Provokationen Webers, sich also vom unpolitischen deutschen Akademikerund Literatentum zu distanzieren, sind mit Warnungen im „Wie ich schon damals sagte"-Stil verbunden. Mit der Schärfe des Ausdrucks, der Betonung der Lächerlichkeiten der Vorschläge und der ins Extreme getriebenen Karikatur der Ansichten versucht Weber, zumindest einige der Betroffenen selbst zum Umdenken zu bringen. Die „einseitige Steigerung” ist nicht nur ein methodisches Prinzip seiner akademischen Schriften, sondern zugleich ein Instrument seiner Rhetorik der Provokation.

\section{Die Neutralisierung eines Schimpfworts: Demagogie}

Die Webersche Praxis, denselben Begriff gelegentlich mit unterschiedlicher normativer Konnotation zu verwenden, macht dem Leser oft Kopfzerbrechen. Die jeweils gemeinte Färbung muss durch den und im jeweiligen Text ausgelegt werden, ohne sicher sein zu können, dass kein ironischer Ton mitschwingt. In einigen Fällen mag es auch so sein, dass Weber erst nach einem abwertenden 
umgangssprachlichen Gebrauch zu einem neutralisierten bzw. aufwertenden Sinn eines Begriffs gelangt.

Dies ist bei der Demagogie bzw. beim Demagogen der Fall. Ich nutze hier die Möglichkeit, mit einer Internetsuche den Gebrauch dieser Ausdrücke systematisch durchzusehen. In der sog. Potsdamer Internetausgabe sind die Sammelbände der „Mariannenausgabe" der zwanziger Jahre auffindbar (http://www.unipotsdam.de/u/paed/Flitner/Flitner/Weber/index.htm). Um die Webersche Begriffspolitik am Beispiel des Demagogen und der Demagogie zu illustrieren und zu spezifizieren, nehme ich die Gesammelten Politischen Schriften als Textbasis.

In dieser Ausgabe verwendet Weber den Ausdruck „Demagog-” 83-mal. Dabei entspricht der Gebrauch oft - besonders in seiner Parlamentsschrift - dem alläglichen pejorativen Wortsinn. Charakteristisch für diesen unreflektierten Gebrauch ist jedoch das Auftreten der Demagogie in Komposita „Admiralsdemagogie”, „'Vaterlands'-Demagogie”, „alldeutsche Demagogie”, „Massendemagogie”, „Straßendemagogie”, „Boulevard-Demagogie” - um nur die auffälligsten Beispiele zu erwähnen.

Diese Ausdrücke sind politisch nach zwei Seiten gerichtet. Die drei letzten Beispiele entsprechen dem alläglichen Ton, der sich - was Weber angesichts der russischen Revolution bis zu einem gewissen Grad als berechtigt ansieht - gegen die Gefahren der Demokratisierung richtet. Aber er parodiert diese Haltung in seiner Parlamentsschrift auch: „Die bei uns populäre Literatenauffassung ist mit der Frage der Wirkung der 'Demokratisierung' schnell fertig: der Demagoge kommt oben auf, und der erfolgreiche Demagoge ist der Mann, der in den Mitteln der Umwerbung der Massen am unbedenklichsten ist." (1918a, 264)

Es ist aber durchaus interessant zu notieren, wie Weber den Vorwurf der Demagogie gegen diejenigen richtet, die eine Polemik gegen die "Massendemagogie" am eifrigsten betrieben: die Admirale, die sog. Vaterlandspartei und die Alldeutschen, nicht zuletzt Wilhelm II selbst (ebenda, bes 249). Demagogie können auch diejenigen betreiben, die am meisten vor Demagogen warnen.

Mehrfach benutzt Weber den Ausdruck Demagoge auch für Bismarck, und hier ist sicherlich der pejorative Wortsinn mitgemeint. Trotzdem geht es nach Weber bei Bismarck auch um eine bestimmte Machttechnik, die - wie sich etwa an folgender Stelle zeigt - an den Demos appelliert: „Demagogie, und zwar eine sehr schlechte Demagogie, wurde in Bismarcks Händen auch die soziale Gesetzgebung des Reiches, so wertvoll man sie rein sachlich finden mag" (ebenda, 210). Die Qualifizierung der Demagogie als „schlecht” verweist schon darauf, dass der Begriff bei Weber durchaus formal verwendbar ist. Ähnliches kann man vom Ausdruck „bloße Demagogie” sagen, der auch auf einen angemessenen Gebrauch des Mittels hinweist, wenn es nicht zur Alleinherrschaft gelangt.

Bei Bismarck bezeichnet Demagogie um einen plebiszitären Politikstil, den man auch aus der amerikanischen Präsidentschaft kennt: „Insbesondere natürlich die 
durch (formell) 'demokratische' Nomination und Wahl legitimierte Machtstellung des Präsidenten der Vereinigten Staaten, dessen Überlegenheit gegenüber dem Parlament eben hierauf beruht. Die Hoffnungen, welche eine so cäsarische Gestalt wie Bismarck an das gleiche Wahlrecht knüpfte, und die Art seiner antiparlamentarischen Demagogie lagen, nur in ihrer Formulierung und Phrase den nun einmal legitimistischen Bedingungen seiner Ministerstellung angepaßt, in der gleichen Richtung " (ebenda, 266). In einem weiten Sinn sind Demokratie und Demagogie für Weber miteinander verbunden: „Demokratisierung und Demagogie gehören zusammen. Aber: ganz unabhängig - das sei wiederholt - von der Art der Staatsverfassung, sofern nur die Massen nicht mehr rein als passives Verwaltungsobjekt behandelt werden können, sondern in ihrer Stellungnahme aktiv irgendwie ins Gewicht fallen." (ebenda, 265).

Aus dieser Sicht wertet Weber den demagogischen Politikstil auf, in dem er eine in Massendemokratien in Frage kommende Alternative zur universellen Tendenz der Bürokratisierung sieht. Demagogie ist für den Politiker im Kampf gegen die Bürokratie notwendig: "(D)aß für die politische Führerschaft jedenfalls nur Persönlichkeiten geschult sind, welche im politischen Kampf ausgelesen sind, weil alle Politik dem Wesen nach Kampf ist. Das leistet nun einmal das vielgeschmähte 'Demagogenhandwerk' im Durchschnitt besser als die Aktenstube, die freilich für sachliche Verwaltung die unendlich überlegene Schulung bietet." (ebenda).

In Politik als Beruf, in dem sein Hauptinteresse auf den Typus des Politikers gerichtet ist, formalisiert Weber seinen Begriffsgebrauch. Der 'große Demagoge' ist ein Typus, für den charismatische Legitimität möglich ist (Weber 1919, 37). Zu den Sondermerkmalen des Okzidents gehört auch der Politiker, dessen Ursprung in den „freien 'Demagogen"” der Antike liegt (ebenda, 38). Den Demagogen Perikles nimmt Weber als Ausgangspunkt seiner Darstellung der historischen Typen des Berufspolitikers: „Der 'Demagoge' ist seit dem Verfassungsstaat und vollends seit der Demokratie der Typus des führenden Politikers im Okzident. Der unangenehme Beigeschmack des Wortes darf nicht vergessen lassen, daß nicht Kleon, sondern Perikles der erste war, der diesen Namen trug." (ebenda, 54).

Im diesem neutralisierten oder gar aufgewerteten Sinn bezeichnet der Demagoge im Allgemeinen einen rhetorischen Politikertypus, den Weber gegen den Beamtentypus stellt. Die Demagogie ist hier insofern wichtig, als die Rede für den Politiker in unterschiedlichen Regimes unverzichtbar ist: „(D)ie Macht der demagogischen Rede (ist) vor allem maßgebend" (ebenda, 65). Der Zusammenhang zwischen Demokratie und Demagogie wird letztlich durch die Rhetorik vermittelt: „Denn die heutige Politik wird nun einmal in hervorragendem Maße in der Öffentlichkeit mit den Mitteln des gesprochenen oder geschriebenen Wortes geführt" (ebenda, 53).

Das Beispiel der Demagogie deutet an, wie Max Weber mit den Bedeutungen von Begriffen spielt. Er schließt sich dem alläglichen Wortgebrauch an, wenn es seinen eigenen Zwecken dient, tut dies aber so, dass er für die naiven und eifrigen Anhänger eines Begriffs Gebrauchsweisen andeutet, die diesen keineswegs einen 
Gefallen tun. Der Demagoge ist auch ein Begriff, mit dem Weber normative Dimensionen öffnet, d.h. die ausschließlich pejorative Verwendung ins Neutrale oder gar ins Lobende umkehrt. Die Neutralisierung ist vor allen ein Medium der Akademisierung der Alltagsbegriffe, und - anstatt neue Termini technici zu konstruieren - eine Erfindung des Potentials dazu. Zwischen dem alltäglichen und dem akademischen Wortgebrauch gibt es bei Weber jedoch keine eindeutige Wasserscheide, sondern eine „range of variation”, wie Quentin Skinner (1979) sagt. In der Sprache Skinners kann man Webers Begriff des Demagogen auch als Modellbeispiel der rhetorischen Umschreibung von Begriffen begreifen (s. Skinner 1996, 2002).

\section{Die Anarchie der Produktion}

Die entscheidende Bedeutung Nietzsches - nicht nur für Webers Religionsanalysen, sondern auch für seine perspektivistische Wissenschaftstheorie - wird in der neueren Literatur (etwa seit Peukert 1989) weitgehend anerkannt. Eines der Schlagwörter Nietzsches, das aus der antiken Rhetorik stammt, ist die Formel der Umwertung der Werte (vgl. Skinner 1996 und 1999 für die Rolle des Schemas paradiastole in der rhetorischen Umwertung der Begriffe).

Diese Strategie gehört explizit zu Webers begriffspolitischem Instrumentarium. Gegen Ende seiner Analyse der Lage der "bürgerlichen Demokratie in Russland” heißt es zum Beispiel: „Was jetzt..., solange die ökonomische und geistige "Revolution”, die vielgeschmähte 'Anarchie' der Produktion und der ebenso vielgeschmähte 'Subjektivismus' noch ungebrochen bestehen, dem durch sie, und nur durch sie, auf sich selbst gestellten Individuum der breiten Massen nicht als 'unveräußerliche' Persönlichkeits- und Freiheitssphäre gewonnen wird, das wird ihm ... vielleicht niemals erobert werden..." (Weber 1906b, 101). Zu beachten ist hier die gegen Marx und die zeitgenössischen Sozialisten gerichtete Aufwertung der "Anarchie der Produktion”. Damit lobt Weber - was zeitgenössische Apologeten des Kapitalismus kaum hätten leisten können - zugleich die Konkurrenzwirtschaft. Wie Weber kurz zuvor sagt: „'Wider dem Strom' der materiellen Konstellation sind wir 'Individualisten' und Parteigänger 'demokratischer' Institutionen” (ebenda 99100).

Man merkt überall die Anführungszeichen. Es ist eben nicht der 'übliche Sinn' der Anarchie, des Individualismus und der Demokratie, den Weber verteidigt. Vielmehr greift er Schimpfworte auf, um sie in seinem eigenen Sinne aufzuwerten und zugleich eine Distanz zu den bekennenden Anarchisten aufzuzeigen, die ja kaum Anhänger einer „Anarchie der Produktion” waren. Noch in der Parlamentsschrift von 1918 wird diese Aufwertung von Anarchie, Individualismus und Demokratie von Weber explizit gegen die Kritik der "Literaten" aufrechterhalten: «Eine "organische", d. h. eine orientalisch-ägyptische Gesellschaftsgliederung, aber im Gegensatz zu dieser so streng rational wie eine 
Maschine es ist, würde dann heraufdämmern. Wer wollte leugnen, daß derartiges als eine Möglichkeit im Schoße der Zukunft liegt? Es ist das schon oft gesagt worden, und die verworrene Vorstellung dieser Möglichkeiten zieht ihre Schatten in die Produktionen unserer Literaten. Nehmen wir nun einmal an: gerade diese Möglichkeit wäre ein unentrinnbares Schicksal, - wer möchte dann nicht lächeln über die Angst unserer Literaten davor, daß die politische und soziale Entwicklung uns künftig zuviel "Individualismus" oder "Demokratie" oder dergleichen bescheren könnte und daß die "wahre Freiheit" erst aufleuchten werde, wenn die jetzige "Anarchie" unserer wirtschaftlichen Produktion und das "Parteigetriebe" unserer Parlamente beseitigt sein werden zugunsten "sozialer Ordnung" und "organischer Gliederung" - das heißt: des Pazifismus der sozialen Ohnmacht unter den Fittichen der einzigen ganz sicher unentfliehbaren Macht: der Bureaukratie in Staat und Wirtschaft! (1918a, 221-222)».

An dieser Stelle deutet Weber die Ähnlichkeiten zwischen dem vorkapitalistischen und dem sozialistischen Gemeinschaftsdenken an. Seine Pointe liegt darin, dass die Denunziation der "Anarchie der Produktion” zugleich den „Individualismus” und die "Demokratie” bedrohen, was zwar viele, nicht aber alle ihrer Gegner auch befürworten.

Im Sozialismus-Aufsatz, als Vortrag 1918 vor Offizieren in Wien gehalten, kommt Weber auf das Schlagwort zurück. Hier legt er den Begriff etwas detaillierter aus: „Diese Privatwirtschaftsordnung hat die sozialistische Theorie mit dem Schlagworte von der "Anarchie der Produktion" belegt, weil sie es darauf ankommen läßt, ob das Eigeninteresse der einzelnen Unternehmer an dem Absatze ihrer Produkte: das Interesse daran, Gewinn zu machen, so funktioniert, daß dadurch eine Versorgung derjenigen, die dieser Güter bedürfen, gewährleistet ist." (Weber 1918b, 310). Zur Erläuterung dessen, was im Sozialismus fehlt, heißt es bei Weber: „(D)ie sogenannte Anarchie der Produktion, d.h. die Konkurrenz der Unternehmer untereinander" (ebenda, 312). Den Offizieren versucht Weber also sichtbar zu machen, dass "Anarchie” nicht immer schlimm sein muss, obwohl man vermuten kann, dass Anarchie für viele Militärs schlimmer war als Sozialismus.

Eine andere Formel Webers, die eine im Vergleich zum bisherigen Gebrauch noch stärkere Degradierung eines Begriffs betreibt, findet man im selben Vortrag. Er bezeichnet die bolschewistische Praxis der Soldatenräte als „eine Militärdiktatur, zwar nicht der Generäle, aber der Korporäle” (ebenda, 323). Zugleich wird die Formel der Diktatur des Proletariats mit dem Begriff der Militärdiktatur verbunden: „Es ist das einzige ganz große Experiment einer "Diktatur des Proletariats«, das bisher gemacht wurde" (ebenda). Mit dieser Zusammenführung zweier Begriffe der Diktatur werden beide nicht nur gegeneinander ausgespielt, sondern auch weiter degradiert.

\section{Politik der Auflösung}


Max Weber widerstrebte schon der Gedanke eines kohärenten Denksystems: „Ein System der Kulturwissenschaften auch nur in dem Sinne einer definitiven, objektiv gültigen, systematisierenden Fixierung der Fragen und Gebiete, von denen sie zu handeln berufen sein sollen, wäre ein Unsinn in sich" (Weber 1904, 184). In diesem Sinne greift er der späteren Begriffsgeschichte vor: „Stets wiederholen sich die Versuche, den "eigentlichen«, "wahren« Sinn historischer Begriffe festzustellen, und niemals gelangen sie zu Ende". (ebenda, 206, s. Palonen 2000, 2003b) Insofern sollen die lexikalischen Quasi-Definitionen der Soziologischen Grundbegriffe auch primär als Instrumente der Kritik im Zeichen der beispielhaften Einführung der Begriffsbildung unter Präsentation alternativer „reinen Typen” verstanden werden.

Es ist auch deutlich, dass Max Weber beansprucht, zentrale Teile des zeitgenössischen Begriffsapparats aufzulösen. Viele seiner wissenschafts- und begriffsgeschichtlichen Bemerkungen - die folgende Charakterisierung der Nationalökonomie etwa - dienen der Auflösung des betreffenden Begriffsapparats: „Aber die Eigenart jener Weltanschauung mit ihrem optimistischen Glauben an die theoretische und praktische Rationalisierbarkeit des Wirklichen wirkte wesentlich insofern, als sie hinderte, daß der problematische Charakter jenes als selbstverständlich vorausgesetzten Gesichtspunktes entdeckt wurde“ (Weber 1904, 185).

Nun konnte Weber aber nicht alles gleichzeitig über Bord werfen. Insofern benutzte er intellektuelle Instrumente mehrerer zeitgenössischer Denkrichtungen, etwa der historischen Schule und der ihr traditionell entgegengesetzten marginalistischen Theorie in der Nationalökonomie oder des Neukantianismus, der Phänomenologie und vor allem das Werk Nietzsches mit seiner Wissenschaftstheorie. Dieser Bezug war jedoch thematisch spezifisch und diente den besonderen Zwecken Webers. Aus dieser Sicht sind seine Zustimmungen eher verdächtig und die direkten Konfrontationen in seinen Pamphleten durchaus deutlich, obwohl er gegenüber seinen Freunden, wie zum Beispiel Naumann, auch dort gelegentlich Höflichkeitsfloskeln prägt.

Aber nach welcher Art von akademischen Machtanteilen strebte Weber mit seinem eigenen Werk? Offensichtlich gab für eine akademische Hegemonie einer neuen Sichtweise à la Weber höchstens marginale Chancen. Ebenso klar ist, dass Weber kaum ein akademischer Mandarin sein wollte. Dagegen zeigen mehrere seiner Polemiken, dass es ihm recht gewesen wäre, bestimmte Ansätze aus dem akademischen Wettbewerb überhaupt auszuschließen. Man denke etwa nur seine Auseinandersetzung mit Wilhelm Ostwalds „energetischen Kulturtheorien”, die Weber aus politischen Gründen gern aus der Diskussion ausschalten wollte: «Denn ein Apostel der "Ordnung" und der Vermeidung "energievergeudenden" Echauffements für andere als technologische Ideale, wie es Ostwald ist und konsequenterweise sein muß, verbreitet - ob er will oder nicht (und wahrscheinlich geschähe dies sehr gegen Ostwalds Willen) - unvermeidlich eine Gesinnung der Fügsamkeit und Anpassung gegenüber den gegebenen sozialen Machtverhältnissen, 
wie sie den matter-of-fact-men aller Epochen gleichmäßig eigentümlich war. Freiheit der Gesinnung ist nun einmal sicherlich kein technologisch oder utilitarisch wertvolles Ideal und "energetisch" nicht begründbar». (Weber 1909, 422-423)

Hier geht es eben nicht um Webers mangelndes Vertrauen in die akademischen Chancen Oswalds, einen eigenen Beitrag zur akademischen Theoriepolitik zu liefern. Vielmehr sind die philosophischen Annahmen Ostwalds nach Webers Ansicht für die politische Freiheit überhaupt katastrophal. Deswegen möchte er ihn aus dem akademischen Betrieb fernhalten. Die Regelung des Theoriestreits enthält auch die Möglichkeit, diejenigen Spieler auszuschließen, die mit unfairen Mitteln spielen, und bei Ostwald ist dies für Weber gerade der Fall. Deswegen ist die Ablehnung auch kategorischer als in seinen sonstigen Polemiken.

Die eigene Perspektive als akademischen Machtanteil kann Weber nur als berechtigte Teilnahme am Theorienstreit behaupten. Das Beispiel Ostwalds verweist darauf, dass dies auch das Recht enthält, zu sagen, wer aus der Konkurrenz ausgeschlossen werden sollte. Ein eigenes Profil zu schaffen, darf nicht zu einer Wissenschaftsauffassung führen, die zur Beendigung jedes Theoriestreits führt, ebenso wie es dem Parlamentarismus nicht erlaubt ist, den Parlamentarismus durch Mehrheitsbeschlüsse abzuschaffen.

\section{Der Wissenschaftler: Beamter oder Politiker?}

Nach konventioneller Ansicht steht der Wissenschaftler für vita contemplativa. Wenn es um die Begriffsbildung geht, ist eine rein kontemplative Haltung für den Wissenschaftler jedoch praktisch unmöglich. Aus der Sicht Webers können Begriffe nie aus dem Gegenstand der Untersuchung entnommen werden: „Wenn immer wieder die Meinung auftritt, jene Gesichtspunkte könnten dem "Stoff selbst entnommen« werden, so entspringt das der naiven Selbsttäuschung des Fachgelehrten, der nicht beachtet, daß er von vornherein kraft der Wertideen, mit denen er unbewußt an den Stoff herangegangen ist, aus einer absoluten Unendlichkeit einen winzigen Bestandteil als das herausgehoben hat, auf dessen Betrachtung es ihm allein ankommt" (Weber 1904, 181).

Wie aber soll sich der Forscher zu diesem Zwang der Stoffauswahl und der Perspektivenbildung verhalten. Meiner These nach hat gibt es zwei historische Idealtypen, die auch als Paradigmen der Forschungspraktiken dienen, nämlich den Beamten und den Politiker: "Der echte Beamte - das ist für die Beurteilung unseres früheren Regimes entscheidend - soll seinem eigentlichen Beruf nach nicht Politik treiben, sondern: "verwalten", unparteiisch vor allem, - auch für die sogenannten "politischen" Verwaltungsbeamten gilt das, offiziell wenigstens, soweit nicht die "Staatsräson", d. h. die Lebensinteressen der herrschenden Ordnung, in Frage stehen. Sine ira et studio, "ohne Zorn und Eingenommenheit" soll er seines Amtes walten. Er soll also gerade das nicht tun, was der Politiker, der Führer sowohl wie seine Gefolgschaft, immer und notwendig tun muß: kämpfen. Denn 
Parteinahme, Kampf, Leidenschaft - ira et studium - sind das Element des Politikers» (Weber 1919, 53).

Mit einer einseitigen Steigerung kann man diese Gegenüberstellung durchaus auf die Forschungspraktiken anwenden. So kann man die Webersche Pointe zu der These ausweiten, dass auch unter den Wissenschaftlern zwei entgegengesetzte Menschentypen zu finden sind, nämlich den Beamtentypus und den Politikertypus. Für Weber sind beide Typen legitim. So wie für ihn die kontinentalen Beamten der Dilettantenverwaltung des amerikanischen spoils system an Effektivität überlegen ist, sind die Normalwissenschaftler in bestimmten spezialisieren akademischen Bereichen den politischen Dilettanten des akademischen Betriebes durchaus überlegen, da diese das Handwerk der Wissenschaften nicht beherrschen. Dagegen reicht der Beamtentypus dort nicht aus, wo die Normen und Kriterien der "Wissenschaft" selbst umstritten sind, wie es für die historischen Wissenschaften typisch ist: "(E)s gibt Wissenschaften, denen ewige Jugendlichkeit beschieden ist, und das sind alle historischen Disziplinen, alle die, denen der ewig fortschreitende Fluß der Kultur stets neue Problemstellungen zuführt. Bei ihnen liegt die Vergänglichkeit aller, aber zugleich die Unvermeidlichkeit immer neuer idealtypischer Konstruktionen im Wesen der Aufgabe." (Weber 1904, 206).

In den historischen Wissenschaften benötigt man eben die Bereitschaft zum Neuen und zur Auflösung des Alten, wie sie dem Politikertypus des Wissenschaftlers eigen ist. Die ewige Jugendlichkeit ist etwas, was dem Beamten verdächtig erscheint, dem Politiker durchaus verständlich und aus eigener Erfahrung, etwa im Sinne des Wiederaufstiegs nach bitteren Niederlagen, durchaus bekannt ist (s. auch Palonen 2005). In diesem Sinne bietet der Politiker durchaus ein Paradigma für einen innovativen Wissenschaftler.

\section{Literaturverzeichnis}

Berolzheimer, Fritz (1907/08): Politik als Wissenschaft. Ihr Wesen und ihre Grenzen. Archiv für Rechts- und Wirtschaftsphilosophie 1, 1907/08, 210-218

Mann, Thomas (1918): Betrachtungen eines Unpolitischen. Frankfurt/M: Fischer 1988.

Mouffe, Chantal (2005): On the Political. London: Routledge.

Palonen, Kari (1998): Das 'Webersche Moment'. Zur Kontingenz des Politischen. Wiesbaden: Westdeutscher Verlag.

Palonen, Kari (2000): Die Umstrittenheit der Begriffe bei Max Weber. Archiv für Begriffsgeschichte-Sonderheft: Die Interdisziplinarität der Begriffesgeschichte, hg. Gunter Scholz. Hamburg: Meiner, 145-158. 
Palonen, Kari (2002): Eine Lobrede für Politiker. Opladen: Leske+Budrich.

Palonen, Kari (2003a): Quentin Skinner. History, Politics. Rhetoric. Cambridge: Polity.

Palonen, Kari (2003b): Die Verzeitlichung der Begriffe bei Max Weber. In: Jussi Kurunmäki \& Kari Palonen (Hg./eds): Zeit, Geschichte und Politik/Time, History and Politik. Jyväskylä: Jyväskylä Studies in Education, Psychology and Social Research 223, 85-103.

Palonen, Kari (2004): Die Entzauberung der Begriffe. Das Umschreiben der politischen Begriffe bei Quentin Skinner und Reinhart Koselleck. Münster: LIT.

Palonen, Kari (2005): Political Theorizing as a Dimension of Political Life. European Journal of Political Theory 4, 351-366.

Peukert, Detlef (1989): Max Webers Diagnose der Moderne. Göttingen: Vandenhoek \& Ruprecht.

Redlich, Josef (1905): Recht und Technik des Englischen Parlamentarismus. Leipzig: Duncker \& Humblot.

Schmitt, Carl (1932): Der Begriff des Politischen. Berlin: Duncker\&Humblot 1979.

Skinner, Quentin (1974): Some Problems in the Analysis of Political Thought and Action. Political Theory 2, 277-303.

Skinner, Quentin (1979): The Idea of a Cultural Lexicon. Essays in Criticism 29, 205-224.

Skinner, Quentin (1996): Reason and Rhetoric in the Philosophy of Hobbes. Cambridge: CUP.

Skinner, Quentin (1999): Rhetoric and Conceptual Change. Finnish Yearbook of Political Thought 3, 60-73.

Skinner, Quentin (2001): Why Laughing Mattered in the Renaissance?, History of Political Thought 22, 418-447.

Skinner, Quentin (2002): Visions of Politics 1-3. Cambridge: CUP. 
Weber, Max (1904): Die 'Objektivität' sozialwissenschaftlicher und sozialpolitischer Erkenntnis. Gesammelte Aufsätze zur Wissenschaftslehre. Tübingen: Mohr 1973, 146-214)

Weber, Max (1906a): Kritische Studien auf dem Gebiet der kulturwissenschaftichen Logik. Gesammelte Aufsätze zur Wissenschaftslehre. Tübingen: Mohr 1973, 215290.

Weber, Max (1906b): Zur Lage der bürgerlichen Demokratie in Rußland. MaxWeber-Studienausgabe I/10, Tübingen: Mohr 1994, 1-104.

Weber, Max (1909): "Energetische" Kulturtheorien. Gesammelte Aufsätze zur Wissenschaftslehre. Tübingen: Mohr 1973, 400-426.

Weber, Max (1910): Antikritisches zum 'Geist' des Kapitalismus. Protestantische Ethik II. Gütersloh: Siebenstern 1978, 149-187.

Weber, Max (1917a): Der Sinn der 'Wertfreiheit' in den sozialen und ökonomischen Wissenschaften. Gesammelte Aufsätze zur Wissenschaftslehre. Tübingen: Mohr 1973, 489-540.

Weber, Max (1917b): Wahlrecht und Demokratie in Deutschland. Max-WeberStudienausgabe I/15. Tübingen: Mohr 1988, 155-189

Weber, Max (1918a): Parlament und Regierung im neugeordneten Deutschland. Max-Weber-Studienausgabe I/15. Tübingen: Mohr 1988, 202-302.

Weber, Max (1918b): Der Sozialismus. Max-Weber-Studienausgabe I/15. Tübingen: Mohr 1988, 303-326.

Weber, Max (1919): Politik als Beruf. Max-Weber-Studienausgabe 1/17. Tübingen: Mohr 1994, 35-88.

Weber, Max (1922): Wirtschaft und Gesellschaft, Hg. Johannes Winckelmann. Tübingen: Mohr 1980.

Weber, Max, Potsdamer Internetsausgabe, http://www.unipotsdam.de/u/paed/Flitner/Flitner/Weber/index.htm 